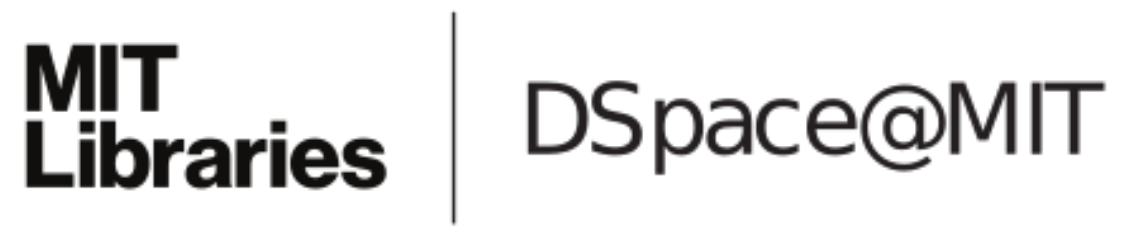

\author{
MIT Open Access Articles
}

A Laplacian characterization of phytoplankton shape

The MIT Faculty has made this article openly available. Please share how this access benefits you. Your story matters.

Citation: Cael, B. B., and Courtenay Strong. "A Laplacian Characterization of Phytoplankton Shape." Journal of Mathematical Biology, vol. 76, no. 6, May 2018, pp. 1327-38.

As Published: http://dx.doi.org/10.1007/s00285-017-1176-8

Publisher: Springer Berlin Heidelberg

Persistent URL: http://hdl.handle.net/1721.1/114907

Version: Author's final manuscript: final author's manuscript post peer review, without publisher's formatting or copy editing

Terms of Use: Article is made available in accordance with the publisher's policy and may be subject to US copyright law. Please refer to the publisher's site for terms of use. 


\title{
A Laplacian characterization of phytoplankton shape
}

\author{
B. B. Cael · Courtenay Strong
}

Received: date / Accepted: date

\begin{abstract}
Phytoplankton exhibit pronounced morphological diversity, impacting a range of processes. Because these impacts are challenging to quantify, however, phytoplankton are often approximated as spheres, and when effects of non-sphericity are studied it is usually experimentally or via geometrical approximations. New methods for quantifying phytoplankton size and shape generally, so all phytoplankton are analyzable by the same procedure, can complement advances in microscopic imagery and automated classification to study the influence of shape in phytoplankton. Here we apply to phytoplankton a technique for defining the size of arbitrary shapes based on the Laplacian - the operator that governs processes, such as nutrient uptake and fluid flow, where phytoplankton shape is expected to have the greatest effect. Deviations from values given by spherical approximation are a measure of phytoplankton shape and indicate the fitness increases for phytoplankton conferred by their non-spherical shapes. Comparison with surface-to-volume quotients suggests the Laplacian-based metric is insensitive to small-scale features which can increase surface area without affecting key processes, but is otherwise closely related to surface-area-to-volume, demonstrating this metric is a meaningful measure. While our analysis herein is limited to axisymmetric phytoplankton due to relative sparsity of 3D information about other phytoplankton shapes, the definition and method are
\end{abstract}

\section{B B Cael}

Massachusetts Institute of Technology

77 Massachusetts Ave

54-1511

Cambridge, MA, 02139, USA

Tel.: +1-401-215-3579

E-mail: snail@mit.edu

Courtenay Strong

Dept. of Atmospheric Sciences

Univ. of Utah

115 Salt Lake City

UT, 84112, USA 
directly generalizable to 3D shape data, which will in the near future be more readily available.

Keywords Phytoplankton $\cdot$ Shape $\cdot$ Size $\cdot$ Laplace's equation

Mathematics Subject Classification (2000) 92B05 · 35J05 - 00A69

\section{Introduction}

Phytoplankton are a key component of the biosphere (Field et al. 1998). As a group, phytoplankton comprise over 5000 known species, displaying a breadth of morphological diversity in both size and shape (Tett and Barton 1995). Shape has a large impact on a range of crucial processes for phytoplankton (Naselli-Flores et al. 2007). Thus, altering shape can increase organism fitness, whether the alteration is increasing aspect ratio, developing appendages, or otherwise.

However, this diversity is challenging to address in full generality, as many phytoplankton shapes are intricate and resist any simple description (Sardet 2015). Effects of phytoplankton shape are typically approached using laboratory experiments (Padisák 2003) or geometrical approximations (Hillebrand et al. 1999). Laboratory experiments directly measure the effect in question but are shape-specific and costly, while geometrical approximations are simple to compute but approximate and limited to a subset of phytoplankton shapes, and require a choice of reference shape. These techniques work well for some applications, but are difficult to generalize to the full range of phytoplankton shapes and processes affected by phytoplankton shape.

In many problems it is common for simplicity to further approximate phytoplankton as spheres, via Equivalent Spherical Diameter (Jennings and Parslow 1988), for which we use the symbol $\ell$. The Equivalent Spherical Diameter of an object is most commonly defined as the diameter of the sphere of equivalent volume to the object; thus it assigns a lengthscale $\ell$ to a plankter by reshaping it. Such approximation removes the capacity to study potential advantages of morphological diversity or drivers of their evolution. It also implicitly assumes volume is the key measure of phytoplankton size, which may not be the case for many aspects of phytoplankton ecology, such as for processes occurring at the interface between the organism and its environment. However, it is occasionally necessary given limited information about many organisms being studied and can be highly convenient. For instance, this lengthscale can be straightforwardly plugged into formulae such as that for the diffusional flux of nutrients to a spherical cell at steady state, $Q=2 \pi \kappa \ell c_{\infty}$, where the flux $Q$ is a function of far-field concentration $c_{\infty}$, diffusivity $\kappa$ and diameter $\ell$ (Karp-Boss et al. 1996). Taking $\ell$ for an irregular object and using such a formula will in general incorrectly compute nutrient flux, often substantially (Karp-Boss and Boss 2015), necessitating the specification of a correcting shape factor. The use of Equivalent Spherical Diameter and a shape factor partitions the influence of size and shape between the two in a coarse sense, though the shape factor may vary not only with phytoplankton shape, but also with the process under consideration and with Equivalent Spherical Diameter itself. 
Extensive research has been conducted into the imaging and classification of phytoplankton. Estep and MacIntyre (1989) developed a system for phytoplankton analysis allowing for counting, sizing, and identifying algae. Culverhouse et al (2001) developed a neural network system for the automated classification of dinoflagellates. Horiuchi et al (2004) developed a continuous imaging system to count and size algae. Rodenacker et al (2006) developed a system to archive digital images of organisms automatically, used for analysis and recognition. Sosik and Olson (2007; Olson and Sosik, 2007) developed a submersible imaging-in-flow instrument and automated classification system for in situ imaging and identification of phytoplankton. Autofluorescence (Hense et al 2008) and image transforms (Kang et al 2009) have been shown to improve organism classification.

These recent developments in two-dimensional imaging technologies provide a wealth of readily available information about phytoplankton shapes for many taxa (Sosik et al. 2015). Confocal microscopy can provide three-dimensional phytoplankton shape information; while these data are presently difficult to acquire, recent developments and cost reductions mean such information is expected to be available in the near future (Roselli et al 2015; Culverhouse 2006); methods have also been proposed to estimate three-dimensional shape information from two-dimensional images (e.g. Moberg and Sosik 2012).

The development of more general metrics of shape, which can be applied to any phytoplankton either simple or complex, can complement these advances in imaging and classification in studying the influence of shape. No one metric will be applicable for all research questions. However, developing metrics of shape related to key processes in phytoplankton dynamics can help assess the increased fitness conferred by phytoplankton shape. Such metrics can complement and aid in the analysis of the data made available by the above-mentioned advances in imaging and classification.

In general, key biological and physical processes in phytoplankton life cycles typically are thought to involve light harvesting, nutrient uptake, and flow - the latter influencing sinking speed, certain predator strategies, and responses to turbulence, among other factors (Naselli-Flores et al. 2007; Vogel 1996; Visser and Jonsson 2000; Padisák et al. 2003). Diffusion has been hypothesized as a driver of phytoplankton morphology (e.g. Young 2006, Sommer 1998). For organisms living at low Sherwood or Peclet number (Cussler 2009), nutrient uptake is governed by diffusive processes, i.e. the balance between time derivatives and the Laplacian of the concentration field $\psi$. At steady state, this balance reduces to

$$
\Delta \psi=0,
$$

which is known as Laplace's equation. Additionally, for flow of an incompressible Newtonian fluid in the limit of small Reynolds number $R e \rightarrow 0$, as is often applicable to local flows near phytoplankton, the Navier-Stokes equations reduce to the Stokes equations (Roland, 2005)

$$
\mu \Delta \mathbf{u}=\nabla p+f
$$

where $\mu$ is the dynamic viscosity of seawater, $\mathbf{u}$ is the local velocity field, $p$ is the local pressure field, and $f$ represents additional forces. Again the Laplacian of the flow field is a key term, and determines the flow in the absence of strong pressure gradients or 
external forces, which are often small in planktonic environments. Sinking, which has long been recognized as playing an important ecological role for phytoplankton (Gran, 1912), is widely modeled using the Stokes equation; just as with diffusive nutrient uptake, cell shape affects sinking rate, necessitating the inclusion of a shape factor when considering non-spherical cells (McKown and Malaika 1950, Walsby and Holland 2006, Lavoie et al 2015). In both cases, improving the estimation and interpretation of shape factors can lead to improvements in the modeling of these key processes.

Here we modify a mathematical technique from other applications (Jones et al, 2000; Strong, 2012) to define a metric for phytoplankton size, which can be used to study the important question of shape in phytoplankton in a new way. This metric may be useful in refining the development and interpretation of shape factors for Laplacian-governed processes. Thus our results are methodological and biological rather than mathematical or physical. We also aim to highlight the need for other such general, process-based metrics of shape for phytoplankton.

\section{Methods}

To quantify the length of an arbitrary shape $P$, one can first solve Laplace's equation within the shape, i.e. using that shape as a domain, specifying Dirichlet boundary conditions of a point source at the centroid $c$ of the shape and zero at the boundary of the shape (Evans, 2010)

$$
\begin{cases}\Delta \psi=0 & \mathbf{x} \in P \backslash c \\ \psi=0 & \mathbf{x} \in \partial P \\ \psi=1 & \mathbf{x}=c .\end{cases}
$$

One should consider an arbitrarily small circle $\varepsilon$ at the centroid of the shape where instead $\left.\psi\right|_{\varepsilon}=1$, as well as a smooth approximation of the boundary of the shape, for analytic well-posedness considerations. Furthermore, the point source $\left.\psi\right|_{c}=1$ should be replaced with a large constant for numerical considerations. We close the Results section with a sensitivity analysis of the placement of the point source at $c$, and a generalization for shapes whose centroids lie outside of $P$.

Solutions to Laplace's equations are called harmonics. Contours of the harmonic $\psi$ will be almost spherical near the centroid of the shape and will deform to take on the shape of $P$; see white curves of Fig. 1c. Then, for each point $p$ on the boundary $\partial P$ there will exist a unique curve $\gamma(p)$ with arc length $|\gamma(p)|$ that follows $\nabla \psi$, i.e. is orthogonal to level sets of $\psi$, connecting that point to the centroid of the shape. The $\gamma$ are called field lines through $\nabla \psi$; see black curves of Fig. 1c. Averaging over all field lines gives a Laplacian-derived lengthscale

$$
\mathscr{L}:=\frac{2}{|\partial P|} \int_{\partial P}|\gamma(p)| d p,
$$

where the factor of 2 is to convert a radius to a diameter and $|\partial P|$ is the area of $\partial P$. This calculation can be performed by numerical integration, as we have done for all results below (scripts available at http://cael.space; all analyses were performed in 
MATLAB, as was the generation of all figures herein). The above definition of $\mathscr{L}$ is a modification of techniques described in Jones et al. (2000) and in Strong (2012); the methods therein was defined for annular regions. Here, replacing the region enclosed by the annulus with a point source, and averaging over the boundary of $P$ allows for a mechanism-motivated computation of $\mathscr{L}$, as phytoplankton are not annular and the processes which motivate the use of the Laplacian (nutrient uptake, interactions with flow) occur at $\partial P$.

This provides a straightforward, objective technique for defining the size of an arbitrary three-dimensional object, without neglecting its nonspherical character, by the Laplacian operator. If we take the body of a plankter as our shape, regions of the plankter's surface with longer associated $\gamma$ field lines will tend to increase the harmonic diameter $\mathscr{L}$, and will also correspond to protrusions or elongations that are known to absorb more nutrients and interact with local flow more strongly (e.g. Nguyen et al., 2011).

If we restrict ourselves to axisymmetric phytoplankton, we can employ the above technique by defining the domain $P$ from a two-dimensional image via rotation, thus taking advantage of a wealth of readily available two-dimensional imagery. In what follows we have used images taken with an Imaging Flow CytoBot (IFCB) (Sosik and Olson 2007; Olson and Sosik 2007) from a manually verified and cataloged database (Sosik et al. 2015), though this procedure should be applicable to any image of a phytoplankton provided that it is axisymmetric and that the lengthscale associated with each pixel is known. Domains were derived from IFCB images using the MATLAB Image Processing Toolbox and the assumption of rotational symmetry; volumes and surface areas for shapes were calculated from these domains by approximating the shapes with conical frustra. Images of all phytoplankton shapes analyzed herein can be found in Fig. 1 and Fig. 2, and URLs from which to retrieve all these images can be found in the Supporting Information.

Plankton are of course three-dimensional, but it is a contemporary challenge to measure accurately their 3D shape. Confocal microscopy (Culverhouse et al. 2006) can provide 3D information to use for $P$, and such information is expected to be widely available soon (Roselli et al. 2015); while the data are difficult to acquire, such information presents an opportunity to extend this work, as do other methods of estimating three-dimensional phytoplankton shape from two-dimensional data (Moberg and Sosik 2012). We emphasize that harmonic diameter is equally computable and tenable for any phytoplankton shape; we focus on axisymmetric shapes here only due to the present difficulty in acquiring 3D information about phytoplankton shape, and because axisymmetric shapes provide an intuitive and defensible example from which to map readily available $2 \mathrm{D}$ information into a $3 \mathrm{D}$ domain. While axisymmetry is itself a geometric approximation, it is a comparatively general one, and made here in order to illustrate the application and computation of $\mathscr{L}$.

To compute $\mathscr{L}$ from a 2D image of an axisymmetric plankter, we reformulate (4) and introduce a weighted integral along the image perimeter $\partial P^{\prime}$, as each perimeter point $p^{\prime}$ on the image corresponds to a half circle with radius equal to the distance $r\left(p^{\prime}\right)$ between the point and the axis of rotation for the phytoplankton. We then instead 
get

$$
\mathscr{L}=\frac{\int_{\partial p^{\prime}} 2 \gamma\left(p^{\prime}\right) \pi r\left(p^{\prime}\right) d p^{\prime}}{\int_{\partial p^{\prime}} \pi r\left(p^{\prime}\right) d p^{\prime}} .
$$

Fig. 1 shows an example of this method for Pleurosigma sp. Our analysis shows a predicted increase in measured size from Equivalent Spherical Diameter, with $\mathscr{L}=$ $201 \mu \mathrm{m}=1.42 \ell$. This increase in size measurement is consistent with the predicted increase in nutrient uptake given by this plankter's aspect ratio (Karp-Boss and Boss 2015 ) if we assume the plankter is approximately spheroidal, suggesting harmonic diameter as a measure of size can incorporate the influence of shape.

\section{Results}

We take a set of manually verified and cataloged IFCB images and compute the harmonic diameter and equivalent spherical diameter for each (see Fig. 2, and Supplementary Materials for a URL from which to access each image). Phytoplankton species were selected to be nearly axisymmetric. As expected, the harmonic diameter was greater than the $\ell$ for each shape, ranging from 1.06 to 3.84 times as large. More elongated phytoplankton having larger $\mathscr{L} / \ell$ ratios is consistent with recen$\mathrm{t}$ work hypothesizing that the interaction between cell shape and diffusive nutrient uptake drives cells towards elongation (Karp-Boss and Boss, 2015).

The surface-to-volume quotient $S / V$ is also thought to be relevant for how phytoplankton interact with their environment (Lewis, 1976). In general, this quotient is an incomplete descriptor of phytoplankton shapes. For instance, shapes which fold to increase surface area dramatically are common in nature, but the rate at which any object can take up nutrients is bounded above by the rate of any sphere that encloses it (Cussler, 2009), even though that sphere may have a much lower $S / V$. However, for phytoplankton without large average curvature such as those considered herein, the $S / V$ appears to be directly related to harmonic diameter. For an arbitrary shape, let $\sigma$ represent the ratio of $S / V$ for the shape and $S / V$ for a sphere of equivalent volume. $\sigma$ in general can be calculated as

$$
\sigma:=\frac{(S / V)_{\text {shape }}}{(S / V)_{\text {sphere }}}=\frac{\ell S_{\text {shape }}}{6 V_{\text {shape }}} .
$$

Both ratios $\mathscr{L} / \ell$ and $\sigma$ are measures of deviation from sphericity, and for a sphere, $\sigma=\mathscr{L} / \ell=1$.

Fig. 3 shows a strong linear correlation (Pearson correlation $r>0.98$ ) for the phytoplankton investigated in Fig. 1 and Fig. 2 between $\mathscr{L} / \ell$ and $\sigma$. This relationship evinces a plausible link between harmonic diameter and surface-to-volume quotient, and points to a simple way to approximate harmonic diameter in the absence of a numerical Laplacian solver. Furthermore, because surface-to-volume quotients are important for both flow and nutrient uptake processes, this tight relationship grounds the nature of the harmonic diameter as a lengthscale for phytoplankton, and demonstrates it is a meaningful measure. This linear correlation between $\sigma$ and $\mathscr{L} / \ell$ is necessarily empirical; we expect additional scatter from investigating additional phytoplankton 
shapes, especially those that are not axisymmetric. Nevertheless, it demonstrates $\sigma$ and $\mathscr{L} / \ell$ are related over a range of axiysmmetric shapes.

Karp-Boss and Boss (2015) also discussed the relative abundance of prolatespheroidal as compared to oblate-spheroidal phytoplankton shapes, due to greater nutrient flux for the same surface area and volume. We observe that the phytoplankton shapes investigated herein are all closer to prolate spheroids based on the relative elongation of their axis of symmetry, and that their $\sigma-\mathscr{L} / \ell$ relationship conforms much more to a prolate-spheroidal one; see Figure 3 . We performed the same analysis described above on ellipses, to compare analyzed phytoplankton shapes to prolate and oblate spheroids. While all regular shapes are restricted to $\sigma=\mathscr{L} / \ell=1$ as they approach sphericity, oblate spheroids' $\sigma$ increases much faster as compared to $\mathscr{L} / \ell$ than either the phytoplankton analyzed here or prolate spheroids. Prolate spheroids follow a logarithmic relationship over the relevant parameter space, with the relationship $\sigma=0.96 \ln (\mathscr{L} / \ell)+(1-0.96 \ln 1)$ accounting for $99.7 \%$ of the variance, and passing through the point $(1,1)$. Plankton with larger $\mathscr{L} / \ell$ values follow this curve closely, while those with lower $\mathscr{L} / \ell$ values reside above the curve, suggesting that small folds and other shape deviations (e.g. Pyramimonas longicauda and Laboea strobila, respectively, Fig. 2) can have a strong impact on surface-to-volume quotients without changing $\mathscr{L} / \ell$ substantially. As such deviations also do not affect nutrient uptake substantially, this distinction favors $\mathscr{L} / \ell$ as a shape factor for nutrient uptake over $\sigma$ for a given $\ell$.

We close the Results section with a sensitivity analysis of the position of the point source within $P$. While plausible, setting the centroid as the location for $\psi=1$ is somewhat arbitrary, and $\mathscr{L}$ may vary with location of point source. Fig. 4 demonstrates this dependence: if $\mathscr{L}_{\mathbf{x}}$ is the harmonic diameter for a point source positioned at $\mathbf{x} \in P$, and $\mathscr{L}_{c}$ is the harmonic diameter with a point source positioned at the centroid of $P$, then $\mathscr{L}_{\mathbf{x}} / \mathscr{L}_{c}$ can exceed 1.3 for a sphere, and exceeds 2.3 for the Pleurosigma sp. shown in Fig. 1. However, in both cases the centroid serves as the global minimum for $\mathscr{L}_{\mathbf{x}}$, and perturbations of the point source off the centroid deviate slowly from $\mathscr{L}_{c}$. Hence the choice of point source location for axisymmetric shapes is not arbitrary. In order to generalize the method to arbitrary 3D shapes, both simple and complex, whose centroid may not lie within $P$, and remove the dependence of $\mathscr{L}$ on the choice of placement of $\mathbf{x}$, we define $\mathscr{L}:=\min _{\mathbf{x} \in P} \mathscr{L}_{\mathbf{x}}$, i.e. the minimum $\mathscr{L}_{\mathbf{x}}$ across all placements of the point source $\mathbf{x} \in P$.

\section{Discussion}

The method presented herein is a generally applicable method for assigning a lengthscale to an arbitrary phytoplankton shape, or equivalently a shape factor (via dividing by $\ell$ ). We have performed this method on several axisymmetric phytoplankton shapes and described how it can be readily generalized to arbitrary phytoplankton shapes. The lengthscale has a qualitative relationship with key processes for phytoplankton, being governed by the same operator. Further investigation is required to make this qualitative relationship precise, and in general the quantitative relationship will vary for different processes. Three-dimensional shape information (Roselli et al 
2015), or another mapping from 2D images to 3D shapes (Moberg and Sosik 2012), is necessary for general phytoplankton shapes that are not rotationally symmetric.

Given 3D shape information for many phytoplankton, generating a database of harmonic diameters or $\mathscr{L} / \ell$ ratios could help implement this technique easily into existing parameterizations that involve phytoplankton size. Solving Laplace's equation in a 3D domain adds no substantial difficulty as compared to 2D solutions, and the weighting factor from the axisymmetric forms we have investigated herein can be dropped. 3D information on phytoplankton shape is preferable for axisymmetric phytoplankton as well; axisymmetry is an approximation we have made herein in order to compute harmonic diameter from 2D data, to illustrate the conceptual utility of the harmonic diameter. While we have only performed calculations on shapes for which this approximation appears reasonable, 3D information does not require such an approximation.

While harmonic diameter formally is computable for any shape, its processedbased motivation is weakened at large enough scales, when the diffusion equation and Stokes flow cease to be the governing equations of the individual in question's environment, i.e. Reynolds, Sherwood, or Peclet numbers cease to be small. Nevertheless, a large number of oceanic organisms of interest live well within this scaling range (Reynolds, 2006). Beyond those discussed herein the method may not be able to address the other factors involved in optimizing shape, e.g. biochemical or buckling constraints; Laplacian-related processes are key drivers in phytoplankton communities, but many factors determine phytoplankton shape (Young, 2006).

Another consideration is that additional information is encoded in the distribution of field line lengths $\gamma$ for a given phytoplankton shape. Herein we only consider the first moment of the distribution to obtain $\mathscr{L}$, but for arbitrary shapes some field lines will be longer than others. A sphere is again a limiting case with zero variance; field lines are of equal length. Higher moments of the distribution may constitute other measures of shape, and in particular may be related to the extent of elongation and appendages.

Even in cases where the harmonic diameter does not significantly deviate from an equivalent spherical estimate, this technique still generalizes a size approximation of phytoplankton to arbitrary shapes in a process-oriented manner. Without adding substantial complexity, harmonic diameter moves beyond the oversimplifying conceptualization of phytoplankton as spheres, or other simple shapes.

Here we have proposed and discussed one metric of phytoplankton shape; we hope to inspire the mathematical investigation into other, complementary or perhaps superior metrics.

Acknowledgements It is a pleasure to thank Heidi Sosik, Lee Karp-Boss, and Emmanuel Boss for invaluable feedback. This research was primarily funded through National Science Foundation awards EPS1208732 and OCE-1315201.

\section{References}

1. Culverhouse PF, Herry V, Reguera B, Gonzalez-Gil S, Williams R, Fonda S, Cabrini M, Parisini T, Ellis R (2001) Dinoflagellate categorisation by artificial neural network (DiCANN). In Harmful algal 

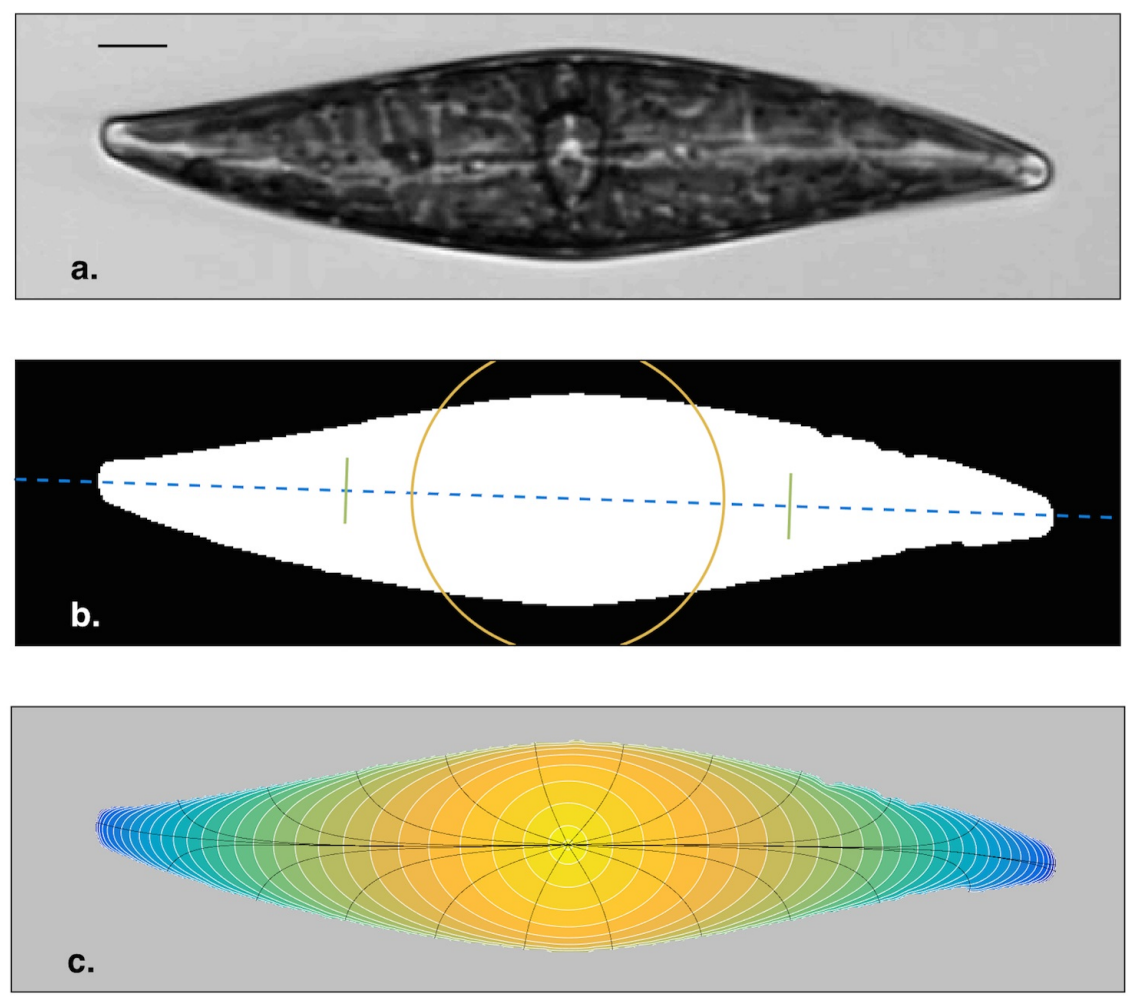

Fig. 1 Example of the analysis method applied to Pleurosigma sp. a) IFCB image of plankter, with $50 \mu \mathrm{m}$ scale bar. All IFCB images have a pixel scale of 0.294 microns. b) image conversion to cross section of domain $P$ (white) for solution of Laplace's equation. Blue dashed line indicates assumed axis of rotation for axisymmetry; yellow circle corresponds to sphere of equivalent volume; green lines demarcate harmonic diameter $\mathscr{L}$.c) solution to Laplace's equation; shading is $\ln \psi$; white lines are $\psi$-contours; black curves are representative field lines $\gamma$. For this planker, $\mathscr{L}=201 \mu \mathrm{m}, \mathscr{L} / \ell=1.42, \sigma=1.55$.

blooms, Hallegraeff GM, Blackburn SI, Bolck CJ, Lewis RJ (eds), Intergovernmental Oceanographic Commission of UNESCO, Vigo, pp 195198

2. Culverhouse PF, Williams R, Benfield M, Flood PR, Sell AF, Mazzocchi MG, Buttino I, and Sieracki M (2006) Automatic image analysis of plankton: future perspectives. Marine Ecology Progress Series 312:297-309

3. Cussler EL (2009) Diffusion: mass transfer in fluid systems. Cambridge university press

4. Estep K and MacIntyre F (1989) Counting, sizing, and identifiaction of algae using image analysis. Sarsia 74.4: 261-268.

5. Evans LC (2010) Partial Differential Equations 2nd ed. American Mathematical Society.

6. Field CB, Behrenfeld MJ, Randerson JT, and Falkowski P (1998) Primary production of the biosphere: integrating terrestrial and oceanic components. Science 281(5374): 237-240

7. Gran HH (1912) Pelagic plant life. In Depths of the Ocean, Murray and Hjort (eds), Macmillan, London, pp 307-386

8. Hense BA, Gais P, Jütting U, Scherb H, and Rodenacker K (2008) Use of fluorsecence information for automated phytoplankton investigation by image analysis. Journal of plankton research 30(5), 587-606

9. Hillebrand H, Dürselen CD, Kirschtel D, Pollingher U, and Zohary T (1999) Biovolume calculation for pelagic and benthic microalgae. Journal of phycology 35(2):403-424 


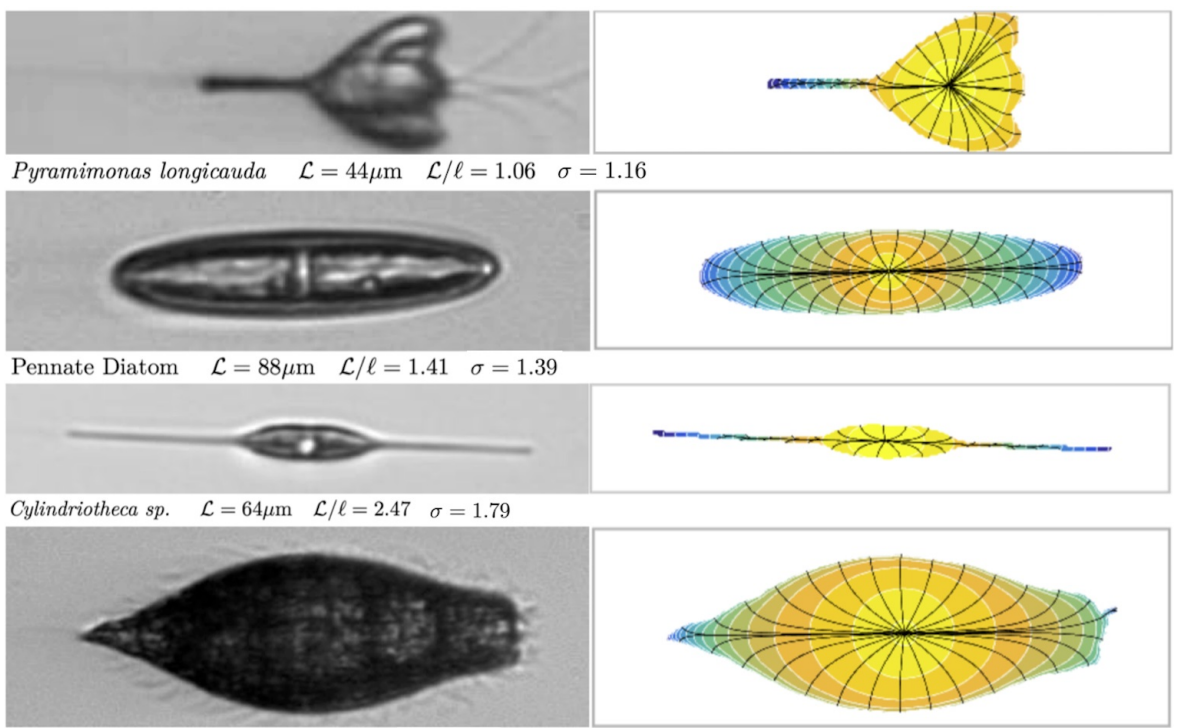

Tiarina fusus $\quad \mathcal{L}=172 \mu \mathrm{m} \quad \mathcal{L} / \ell=1.16 \quad \sigma=1.25$

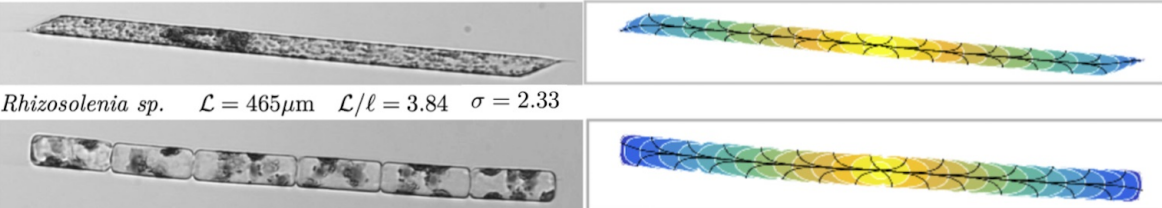

Guardinia delicatula (chain) $\quad \mathcal{L}=464 \mu \mathrm{m} \quad \mathcal{L} / \ell=3.08 \quad \sigma=2.09$

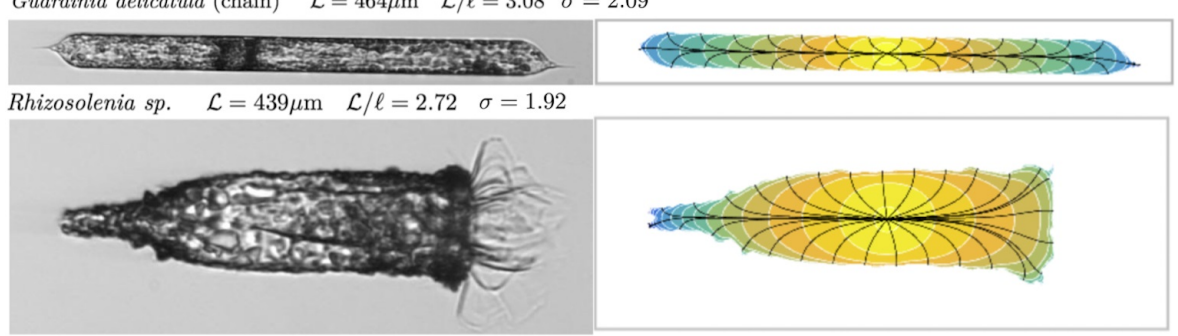

Tinnitid $\quad \mathcal{L}=242 \mu \mathrm{m} \quad \mathcal{L} / \ell=1.43 \quad \sigma=1.48$
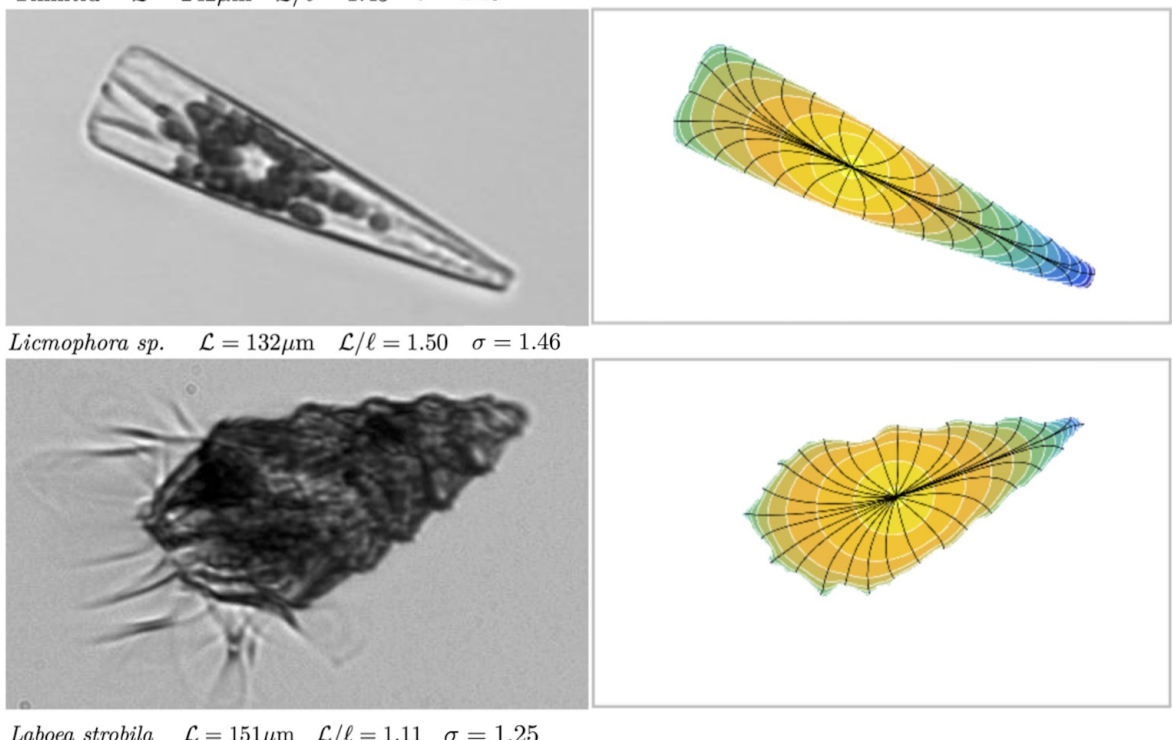

Fig. 2 Examples of the analysis method applied to additional phytoplankton. Left column is IFCB images, resized to have the same horizontal extent; right column is solutions $\psi$ formatted as in Fig. 1; identification, $\mathscr{L}, \mathscr{L} / \ell$, and $\sigma$ reported below each. 


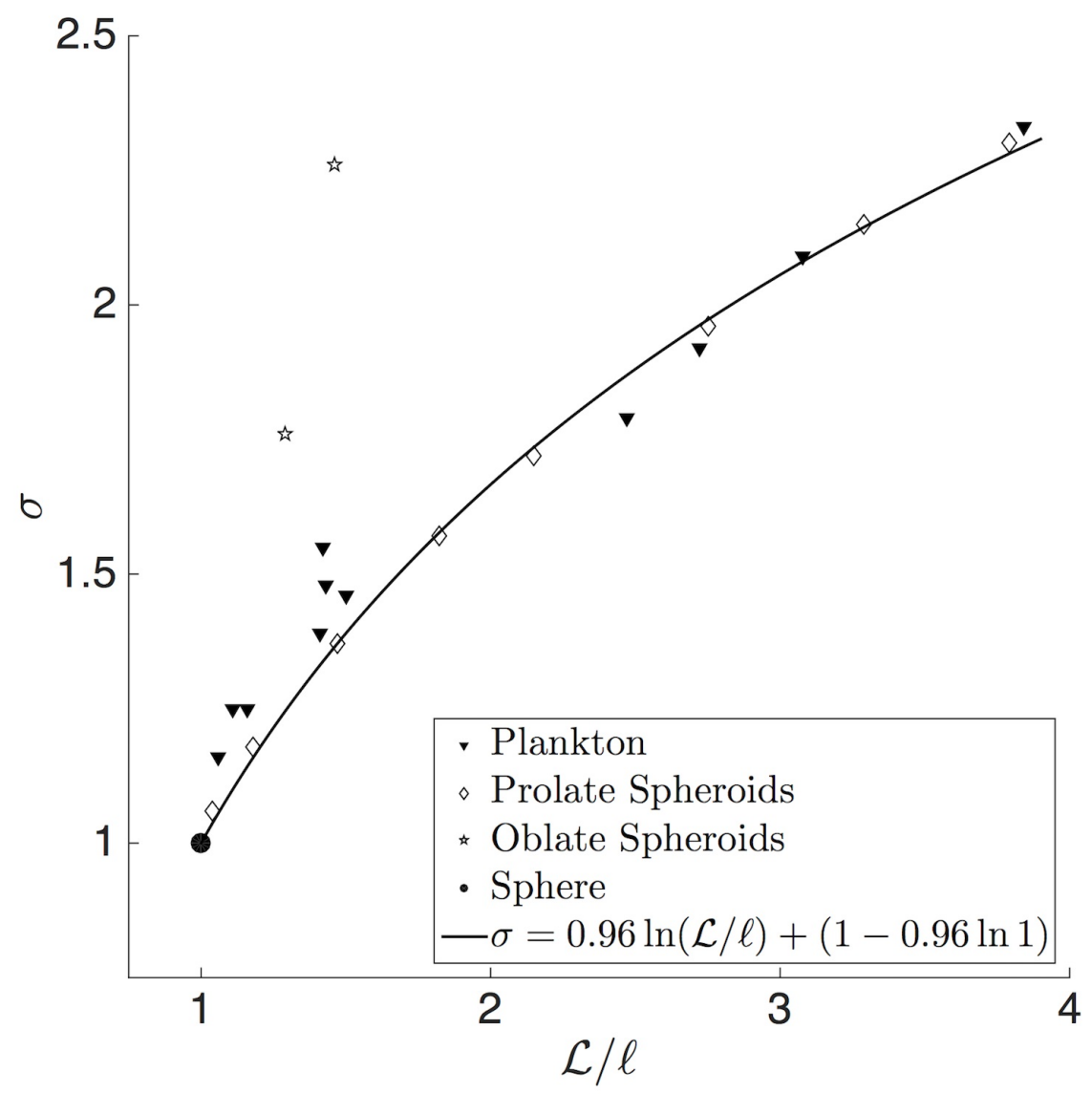

Fig. 3 Scatter plot of $\mathscr{L} / \ell$ (i.e. the ratio of harmonic and equivalent spherical diameters) vs. $\sigma$ (i.e. ratio of surface-to-volume for phytoplankton and their equivalent spheres). Triangles are phytoplankton from Figs. 1, 2; diamonds are prolate spheroids; stars are oblate spheroids; circle is a sphere; solid curve is a 1-parameter logarithmic fit to the prolate spheroid data. OLS linear regression (type I) accounts for $97.0 \%$ of the variance in phytoplankton values, while the above fit accounds for $99.7 \%$ of variance in prolate spheroid values.

10. Horiuchi T, Akiba T, and Kakui Y (2004) Development of a continuous imaging system equipped with fluorescent imaging for classification of phytoplankton. In MTTS/IEEE TECHNO-OCEAN 'O4 3, 1410-1413

11. Jennings BR and Parslow K (1988) Particle size measurement: the equivalent spherical diameter Proceedings of the Royal Society of London A: Mathematical, Physical and Engineering Sciences 419(1856): 137-149

12. Jones SE, Buchbinder BR, Aharon I (2000) Three-dimensional mapping of cortical thickness using Laplaces equation. Hum. Brain Mapp. 11:12-32

13. Kang L, Yang C, and Gao Y (2009) Improved shape description using radon transform and application in phytoplankton identification. In 2nd IEEE International Conference on Broadband Network \& Multimedia Technology, 477-481

14. Karp-Boss L, Boss E, and Jumars PA (1996) Nutrient fluxes to planktonic osmotrophs in the presence of fluid motion. Oceanography and Marine Biology 34:71-108 

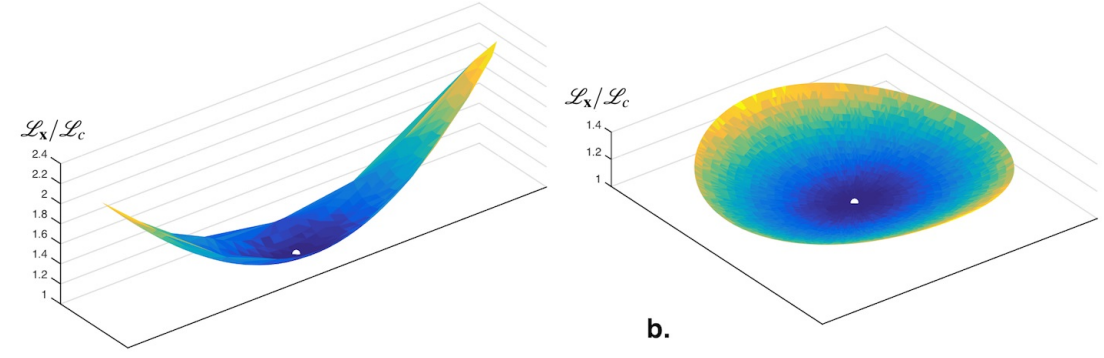

a.

Fig. 4 Sensitivity analysis of point source location. The $z$-value at each horizontal point indicates $\mathscr{L}_{x} / \mathscr{L}_{c}$, i.e. the ratio of harmonic diameter computed when the point source is at that location, versus when the point source is at the centroid. a) Result for Pleurosigma sp. image from Fig. 1a, b) result for a sphere. Note that the results for the sphere depend on the choice of axis of rotation.

15. Karp-Boss L and Boss E (2015) The elongated, the squat and the spherical: selective pressures for phytoplankton shape. In Aquatic microbial ecology and biogeochemistry: a dual perspective, Gilbert PM and Kana TM (eds), in press.

16. Lavoie M, Levasseur M, and Babin M (2015) Testing the potential ballast role for dimethylsulfoniopropionate in marine phytoplankton: A modeling study. Journal of plankton research 37(4): 699-711

17. Lewis W. M. (1976) Surface/volume ratio: implications for phytoplankton morphology. Science 192:885-887

18. McKown JS and Malaika J (1950) Effect of particle shape on settling velocity at low Reynols numbers. Transactions of the American Geophysical Union, 31, 74-82

19. Moberg E and Sosik H (2012) Distance maps to estimate cell volume from two-dimensional plankton images. Limnology and Oceanography: Methods 10:278-288

20. Naselli-Flores L, Padisák J, and Albay M (2007) Shape and size in phytoplankton ecology: do they matter? Hydrobiologia 578(1):157-161

21. Nguyen HV, Karp-Boss L, Jumars PA, and Fauci L (2011) Hydrodynamic effects of spines: a different spin. Limnology and Oceanography: Fluids and Environments 1:110-119

22. Olson RJ and Sosik HM (2007) A submersible imaging-in-flow instrument to analyze nano-and microplankton: Imaging FlowCytobot. Limnology and Oceanography: Methods 5(6):195-203

23. Padisák J, Soróczki-Pintér É, and Rezner Z (2003) Sinking properties of some phytoplankton shapes and the relation of form resistance to morphological diversity of plankton-an experimental study. Aquatic Biodiversity (pp. 243-257)

24. Reynolds CS (2006) The ecology of phytoplankton. Cambridge University Press

25. Roland C, Grace JR, and Weber ME (2005) Bubbles, drops, and particles. Courier 375 Corporation

26. Rodenacker K, Hense B, Gais P (2006). Automatic analysis of aqueous specimens for phytoplankton structure recognition and population esetimation. Microscopy research and technique 69.9: 708-720

27. Roselli L, Paparella F, Stanca E, Basset A (2015) New datadriven method from 3D confocal microscopy for calculating phytoplankton cell biovolume. Journal of microscopy 258(3):200-11

28. Sardet C (2015) Plankton: Wonders of the Drifting World. University of Chicago press.

29. Sommer U (1998) Silicate and the functional geometry of marine phytoplankton. Journal of plankton research 20(9): 1853-1859

30. Sosik HM and Olson RJ (2007) Automated taxonomic classification of phytoplankton sampled with imaging-in-flow cytometry. Limnology and Oceanography: Methods 5(6):204-216

31. Strong C (2012) Atmospheric influence on Arctic marginal ice zone position and width in the Atlantic sector, February-April 1979-2010. Climate dynamics, 39(12):3091-3102

32. Tett P and Barton ED (1995) Why are there about 5000 species of phytoplankton in the sea? Journal of Plankton Research 17(8), 1693-1704

33. Visser AW and Jonsson PR (2000) On the reorientation of non-spherical prey particles in a feeding current. Journal of plankton research 22(4):761-777 
34. Vogel S (1996) Life in moving fluids: the physical biology of flow. Princeton University Press

35. Walsby AE and Holland DP (2006) Sinking velocities of phytoplankton measured on a stable density gradient by laser scanning. Journal of the royal society interface 3, 429-439.

36. Young KD (2006) The selective value of bacterial shape. Microbiology and Molecular Biology Reviews 70(3):660-703 